\title{
ON ONE PROPERTY OF POSITIVE-DEFINITE QUADRATIC FORMS CORRESPONDING TO FINITE POSETS
}

\author{
M. V. Stepochkina
}

UDC 512.647.2+512.562

\begin{abstract}
We consider the Bondarenko hypothesis about the structure of finite posets with positive-definite Tits quadratic form. We prove that the hypothesis holds for a fixed natural number $k>8$ if it holds for the number $k-1$.
\end{abstract}

Quadratic forms are encountered in the solution of numerous problems in algebra, the theory of differential and integral equations, functional analysis, and other fields of mathematics (see, e.g., [1] and references therein; see also [2-17]). Among quadratic forms, an important role is played by Tits quadratic forms for various objects (graphs, posets, algebras, etc.). In the present paper, we study exactly these forms.

In [18], Gabriel associated a quiver (an oriented graph) with a certain quadratic form and called it a Tits quadratic form. This form plays an important role in the theory of finite-dimensional representations of graphs. In particular, it was shown in [18] that a graph has a finite type (i.e., it has a finite, up to an isomorphism, number of indecomposable representations) if and only if the corresponding Tits form is positive definite. This paper originated a new trend in algebra that studies the relationship between the properties of representations of various objects and the properties of the corresponding quadratic forms.

The next step in this direction was made in the works of Brenner [19] and Drozd [20], where Tits quadratic forms were determined for quivers with relations and for posets, respectively. In the general case, the Tits form for matrix problems without relations was introduced by Kleiner and Roiter in [21].

It was shown in [20] that a poset has a finite type if and only if its Tits form is weakly positive (representations of posets were introduced in [22]). Positive-definite forms are not distinguished in this case, but in the further investigation of these representations positive-definite Tits forms play an important role [23]. For the first time, these forms were described in [1] (see also [24]), where infinite posets were considered. In [25], Bondarenko considered the case of finite sets and formulated a hypothesis concerning positive-definite Tits forms. This hypothesis is considered in the present paper.

\section{Formulation of a Hypothesis}

In the present paper, we consider finite posets. A subset $X$ of a poset $S$ is always understood as a subset complete with respect to the partial-order condition (i.e., if $a, b \in X$, then $a \geq b$ in $X$ if and only if $a \geq b$ in $S$ ).

If a poset $S$ is the union of its pairwise disjoint subsets $A_{1}, \ldots, A_{s}, s \geq 1$, then we say that $S$ is the sum of these subsets and write $S=A_{1}+\ldots+A_{s}$. If elements of different summands are always incomparable, then $S$ is called the direct sum of these subsets.

We also recall several definitions from [1].

Let a poset $S$ be the sum of subsets $A_{1}, \ldots, A_{s}$. This sum is called one-sided if (up to reenumeration of summands) one has $i<j$ whenever there exist elements $b \in A_{i}$ and $c \in A_{j}, i \neq j$, such that $b<c$. Further, the sum $S=A_{1}+\ldots+A_{s}$ is called minimax if $x$ is the minimum element and $y$ is the maximum element of the set $S$ whenever $x$ and $y$ belong to different summands and, moreover, $x<y$. Formally, a direct sum of subsets is minimax. However, in what follows, speaking of a minimax sum, we always assume that this sum is not direct.

Shevchenko Kyiv National University, Kyiv.

Translated from Neliniini Kolyvannya, Vol. 8, No. 4, pp. 544-552, October-December, 2005. Original article submitted August $18,2005$. 
A poset with a unique pair of incomparable elements is called an almost chain poset (any linearly ordered set is called a chain set).

Finally, recall the definition of Tits quadratic form $q_{S}(z)$ for an arbitrary poset $S$. By definition, this is a quadratic form $q(z): \mathbb{Z}_{0}^{S \cup 0} \rightarrow \mathbb{Z}$ given by the equality

$$
q(z)=z_{0}^{2}+\sum_{i \in S} z_{i}^{2}+\sum_{i<j, i, j \in S} z_{i} z_{j}-z_{0} \sum_{i \in S} z_{i}
$$

We now formulate the hypothesis mentioned in the introduction (note that, in the first two conditions, empty chain sets are also admissible).

Bondarenko Hypothesis. If $S$ is a poset of order $n \geq 8$ with positive-definite Tits form, then one of the following conditions is satisfied:

(1) $S$ is a direct sum of two chain subsets;

(2) $S$ is a one-sided minimax sum of two chain subsets;

(3) S is a direct sum of a chain subset and an almost chain subset.

Geometrically, the posets indicated in the conditions of the hypothesis have the following form:

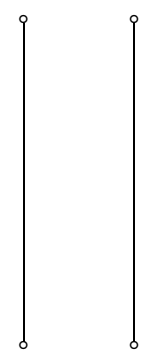

(2)

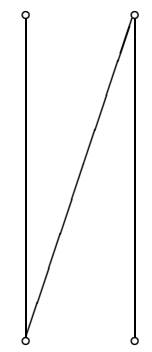

(3)

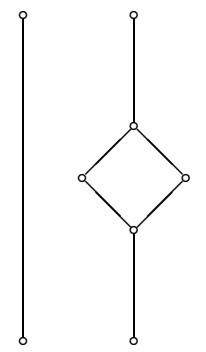

(each vertical segment here is a chain of length $d \geq 0$, and the inclined segments do not contain intermediate points).

Note that the condition $n \geq 8$ is essential. For example, for the poset $T=\left\{a_{1}<a_{2}<a_{3}<a_{4}, a_{5}<a_{6}<\right.$ $\left.a_{7}, a_{1}<a_{6}\right\}$ of order 7 , which satisfies none of conditions (1)-(3), the Tits form is positive definite.

Also note that all posets of the form (1)-(3) have a positive-definite Tits form (see [1]).

The motivation for the formulated hypothesis was also given in [25]. Here we only note that an analogous statement for infinite posets (with certain natural modifications of the definitions presented above) was proved in [1]; furthermore, it follows from a fairly complicated proof of this statement that there exists a certain natural number $N$ such that the hypothesis is true for any $n \geq N$.

\section{Main Result}

The aim of the present paper is to prove the following theorem:

Theorem 1. Suppose that $n \geq 8$ is a natural number for which the Bondarenko hypothesis is true, i.e., any poset of order $n$ with positive-definite Tits form satisfies one of conditions (1)-(3). Then this hypothesis is also true for each natural number $m>n$.

The theorem obviously follows from the purely combinatorial statement presented below. 
Proposition 1. Let $S$ be a poset of order $n>8$ such that every proper subset of it satisfies one of conditions (1)-(3). Then one of these conditions is also satisfied for the set $S$ itself.

Note that the condition presented in the first part of Proposition 1 is satisfied if and only if it is satisfied for subsets of order $n$. In addition, the condition $n>8$ in the statement of the proposition is essential. For example, for every proper subset of the poset (of order 5) $S=\left\{a_{2}<a_{3}<a_{4}<a_{5}, a_{1}<a_{4}\right\}$, one of conditions (1)-(3) is satisfied, whereas the set $S$ does not satisfy any of these conditions (here and in what follows, specifying an individual poset, we define the partial order up to transitivity).

Proof of Proposition 1. First, we introduce several definitions.

Let $\mathcal{P}_{i}$ denote the collection of all posets of the form $(i)$, where $i \in\{1,2,3\}$. We set $\mathcal{P}=\mathcal{P}_{1} \bigcup \mathcal{P}_{2} \bigcup \mathcal{P}_{3}$. Let $\overline{\mathcal{P}}$ denote the collection of all posets that do not belong to $\mathcal{P}$. Specifying individual posets, we define the partial-order relation up to transitivity. Denote the partial-order relation on $S$ by $\prec(<$ denotes the linear-order relation on the set of integers).

We now pass directly to the proof of Proposition 1, which, for clarity, is accompanied by geometric explanations.

We fix a certain maximum point in $S$, denote it by $x_{0}$, and set $T=S \backslash x_{0}$. By virtue of the conditions of the proposition, $T$ has the form (1), (2), or (3).

I. First, consider the case where the poset $T$ has the form (1), namely,

$$
T_{1}=\left\{a_{1} \prec a_{2} \prec \ldots \prec a_{p}, b_{1} \prec b_{2} \prec \ldots \prec b_{q}\right\},
$$

where $p+q=n-1 \geq 8$ (in this case, we have $p \geq 4, q \geq 0$ or $q \geq 4, p \geq 0$ ).

It is easy to see that, for the poset $S$, one of the following cases is realized:

(1.1) $S_{1.1}=\left\{a_{1} \prec a_{2} \prec \ldots \prec a_{p}, b_{1} \prec b_{2} \prec \ldots \prec b_{q}, x_{0}\right\}$ (i.e., $x_{0}$ is a point incomparable with any point of the set $T)$;

(1.2) $S_{1.2}=\left\{a_{1} \prec a_{2} \prec \ldots \prec a_{p}, b_{1} \prec b_{2} \prec \ldots \prec b_{q}, a_{r} \prec x_{0}\right\}$, where $1 \leq r \leq p$;

(1.3) $S_{1.3}=\left\{a_{1} \prec a_{2} \prec \ldots \prec a_{p}, b_{1} \prec b_{2} \prec \ldots \prec b_{q}, a_{r} \prec x_{0}, b_{s} \prec x_{0}\right\}$, where $1 \leq r \leq p$ and $1 \leq s \leq q$

These sets have the following geometric representation:

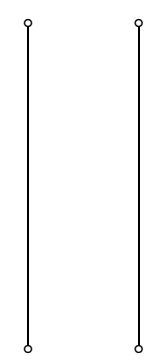

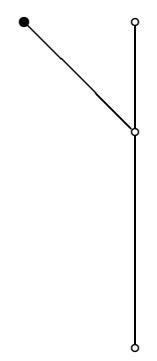

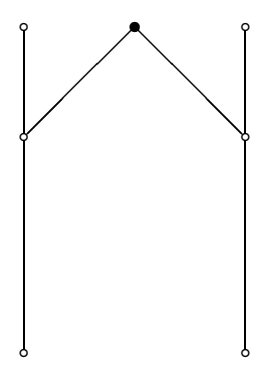

Here and in what follows, for simplicity, we indicate only main points. The numbers of these points are not indicated, but they can easily be determined. The point $x_{0}$ in the figures is marked by $\bullet$.

Consider each of cases (1.1)-(1.3) separately. In the figures, the chains $A=\left\{a_{1} \prec a_{2} \prec \ldots \prec a_{p}\right\}$ and $B=\left\{b_{1} \prec b_{2} \prec \ldots \prec b_{q}\right\}$ are located to the left and to the right, respectively. 
(1.1) For $p=0$ or $q=0$, we obtain case (1). For $p=1$ or $q=1$, we have case (3). In the other cases, if $p \geq 4$, then we delete the point $a_{p}$. If $q \geq 4$, then we delete the point $b_{q}$. In both cases, we obtain a subset of $n-1$ points that belongs to $\overline{\mathcal{P}}$, which contradicts the conditions of the proposition.

(1.2) For $r=p$, we obtain case (1). For $r=p-1$, we have case (3). Let $r \neq p-1, p$. If, in addition, $q=0$ and $r=1$, then we have case (2). If $q=0$ and $r \neq 1$, then we delete the point $a_{3}$. If $q \neq 0$ and $p \geq 4$, then we delete the point $a_{2}$. If $q \geq 4$, then we delete the point $b_{q}$. In each case, we obtain a subset of $n-1$ points that belongs to $\overline{\mathcal{P}}$, which contradicts the conditions of the proposition.

(1.3) If $r=p, s=1$ or $r=1, s=q$, then we obtain case (2). In the other cases, if $r \geq 3$, then we delete the point $a_{r}$. If $s \geq 3$, then we delete the point $b_{s}$. If $r<3, s<3$, and $p \geq 4$, then we delete the point $a_{p}$. If $r<3, s<3$, and $q \geq 4$, then we delete the point $b_{q}$. In each of these four cases, we obtain a subset of $n-1$ points that belongs to $\overline{\mathcal{P}}$, which contradicts the conditions of the proposition.

II. Consider the case where the poset $T$ has the form (2), namely,

$$
T_{2}=\left\{a_{1} \prec a_{2} \prec \ldots \prec a_{p}, b_{1} \prec b_{2} \prec \ldots \prec b_{q}, a_{1} \prec b_{q}\right\}
$$

where $p+q=n-1 \geq 8$ (in this case, we have $p \geq 4$ or $q \geq 4$ ).

It is easy to see that one of the following cases is realized for the poset $S$ :

(2.1) $S_{2.1}=\left\{a_{1} \prec a_{2} \prec \ldots \prec a_{p}, b_{1} \prec b_{2} \prec \ldots \prec b_{q}, a_{1} \prec b_{q}, x_{0}\right\}$;

(2.2) $S_{2.2}=\left\{a_{1} \prec a_{2} \prec \ldots \prec a_{p}, b_{1} \prec b_{2} \prec \ldots \prec b_{q}, a_{1} \prec b_{q}, a_{r} \prec x_{0}\right\}$, where $1 \leq r \leq p$;

(2.3) $S_{2.3}=\left\{a_{1} \prec a_{2} \prec \ldots \prec a_{p}, b_{1} \prec b_{2} \prec \ldots \prec b_{q}, a_{1} \prec b_{q}, b_{r} \prec x_{0}\right\}$, where $1 \leq r \leq q$;

(2.4) $S_{2.4}=\left\{a_{1} \prec a_{2} \prec \ldots \prec a_{p}, b_{1} \prec b_{2} \prec \ldots \prec b_{q}, a_{1} \prec b_{q}, a_{r} \prec x_{0}, b_{s} \prec x_{0}\right\}$, where $1 \leq r \leq p$, $1 \leq s \leq q$, and $(r, s) \neq(1, q)$.

These sets have the following geometric representation:

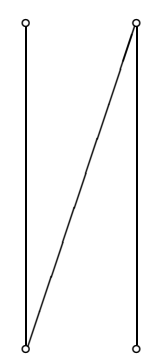

(2.3)

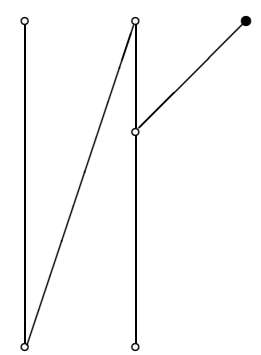

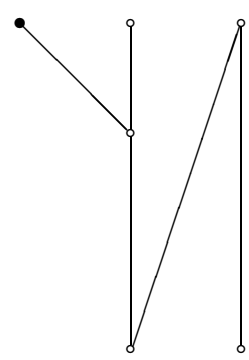

(2.4)

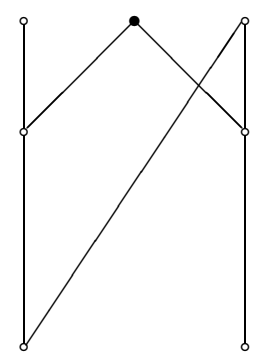

We consider each of cases (2.1)-(2.4) separately. In the figures, the chains $A=\left\{a_{1} \prec a_{2} \prec \ldots \prec a_{p}\right\}$ and $B=\left\{b_{1} \prec b_{2} \prec \ldots \prec b_{q}\right\}$ are located to the left and to the right, respectively. 
(2.1), (2.3) If $p \geq 4$, then we delete the point $a_{p}$. If $q \geq 4$, then we delete the point $b_{1}$. In both cases, we obtain a subset of $n-1$ points that belongs to $\overline{\mathcal{P}}$, which contradicts the conditions of the proposition.

(2.2) For $r=p$, we have case (2). Let $r \neq p$. If $q>1$, then we delete the point $b_{1}$. If $q=1$, then we delete the point $a_{2}$. In both cases, we obtain a subset of $n-1$ points that belongs to $\overline{\mathcal{P}}$, which contradicts the conditions of the proposition.

(2.4) If $p \geq 4$, then we delete the point $a_{p}$. If $q \geq 4$, then we delete the point $b_{2}$. In both cases, we obtain a subset of $n-1$ points that belongs to $\overline{\mathcal{P}}$, which contradicts the conditions of the proposition.

III. Finally, consider the case where the poset $T$ has the form (3), namely,

$$
T_{3}=\left\{a_{1} \prec a_{2} \prec \ldots \prec a_{p} \prec a \prec b_{1} \prec b_{2} \prec \ldots \prec b_{q}, a_{p} \prec b \prec b_{1}, c_{1} \prec c_{2} \prec \ldots \prec c_{r}\right\}
$$

where $p+q+r=n-1 \geq 8$ (in this case, we have $p \geq 2, q \geq 2$ or $r \geq 2$ ).

It is easy to see that one of the following cases is realized for the poset $S$ :

(3.1) $S_{3.1}=\left\{a_{1} \prec a_{2} \prec \ldots \prec a_{p} \prec a \prec b_{1} \prec b_{2} \prec \ldots \prec b_{q}, a_{p} \prec b \prec b_{1}, c_{1} \prec c_{2} \prec \ldots \prec c_{r}, x_{0}\right\}$;

(3.2) $S_{3.2}=\left\{a_{1} \prec a_{2} \prec \ldots \prec a_{p} \prec a \prec b_{1} \prec b_{2} \prec \ldots \prec b_{q}, a_{p} \prec b \prec b_{1}, c_{1} \prec c_{2} \prec \ldots \prec c_{r}, a_{s} \prec x_{0}\right\}$, where $1 \leq s \leq p$;

(3.3) $S_{3.3}=\left\{a_{1} \prec a_{2} \prec \ldots \prec a_{p} \prec a \prec b_{1} \prec b_{2} \prec \ldots \prec b_{q}, a_{p} \prec b \prec b_{1}, c_{1} \prec c_{2} \prec \ldots \prec c_{r}, a \prec x_{0}\right\}$;

(3.4) $S_{3.4}=\left\{a_{1} \prec a_{2} \prec \ldots \prec a_{p} \prec a \prec b_{1} \prec b_{2} \prec \ldots \prec b_{q}, a_{p} \prec b \prec b_{1}, c_{1} \prec c_{2} \prec \ldots \prec c_{r}, b_{s} \prec x_{0}\right\}$, where $1 \leq s \leq q$;

(3.5) $S_{3.5}=\left\{a_{1} \prec a_{2} \prec \ldots \prec a_{p} \prec a \prec b_{1} \prec b_{2} \prec \ldots \prec b_{q}, a_{p} \prec b \prec b_{1}, c_{1} \prec c_{2} \prec \ldots \prec c_{r}, c_{s} \prec x_{0}\right\}$, where $1 \leq s \leq r$;

(3.6) $S_{3.6}=\left\{a_{1} \prec a_{2} \prec \ldots \prec a_{p} \prec a \prec b_{1} \prec b_{2} \prec \ldots \prec b_{q}, a_{p} \prec b \prec b_{1}, c_{1} \prec c_{2} \prec \ldots \prec c_{r}, a \prec x_{0}\right.$, $\left.b \prec x_{0}\right\}$;

(3.7) $S_{3.7}=\left\{a_{1} \prec a_{2} \prec \ldots \prec a_{p} \prec a \prec b_{1} \prec b_{2} \prec \ldots \prec b_{q}, a_{p} \prec b \prec b_{1}, c_{1} \prec c_{2} \prec \ldots \prec c_{r}\right.$, $\left.a_{s} \prec x_{0}, c_{t} \prec x_{0}\right\}$, where $1 \leq s \leq p$ and $1 \leq t \leq r$;

(3.8) $S_{3.8}=\left\{a_{1} \prec a_{2} \prec \ldots \prec a_{p} \prec a \prec b_{1} \prec b_{2} \prec \ldots \prec b_{q}, a_{p} \prec b \prec b_{1}, c_{1} \prec c_{2} \prec \ldots \prec c_{r}\right.$, $\left.b \prec x_{0}, c_{s} \prec x_{0}\right\}$, where $1 \leq s \leq r$;

(3.9) $S_{3.9}=\left\{a_{1} \prec a_{2} \prec \ldots \prec a_{p} \prec a \prec b_{1} \prec b_{2} \prec \ldots \prec b_{q}, a_{p} \prec b \prec b_{1}, c_{1} \prec c_{2} \prec \ldots \prec c_{r}\right.$, $\left.b_{s} \prec x_{0}, c_{t} \prec x_{0}\right\}$, where $1 \leq s \leq q$ and $1 \leq t \leq r$;

(3.10) $S_{3.10}=\left\{a_{1} \prec a_{2} \prec \ldots \prec a_{p} \prec a \prec b_{1} \prec b_{2} \prec \ldots \prec b_{q}, a_{p} \prec b \prec b_{1}, c_{1} \prec c_{2} \prec \ldots \prec c_{r}\right.$, $\left.a \prec x_{0}, b \prec x_{0}, c_{s} \prec x_{0}\right\}$, where $1 \leq s \leq r$.

These sets have the following geometric representation:
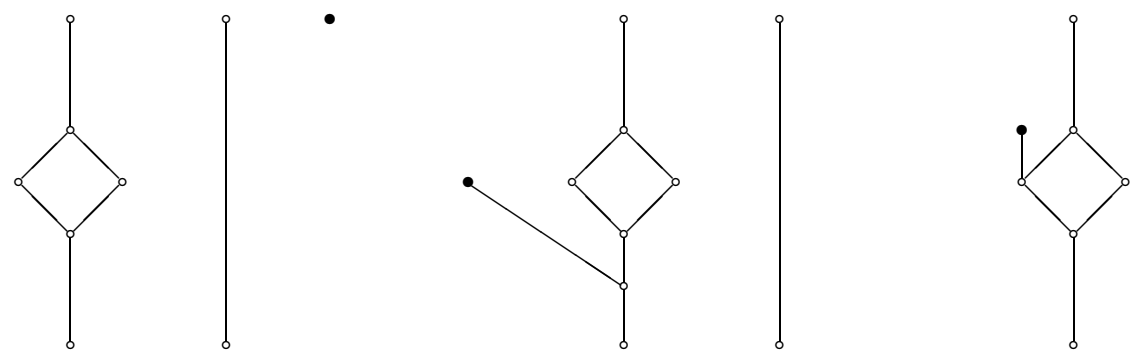

$$
\text { i }
$$




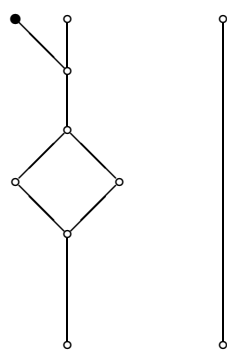

(3.7)

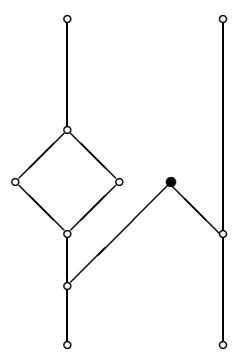

(3.5)

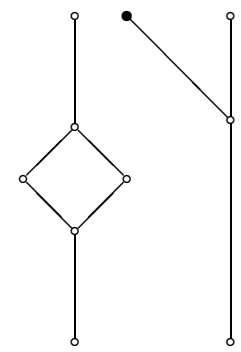

$(3.8)$

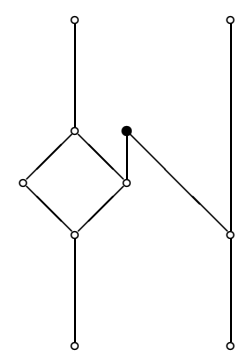

(3.10)

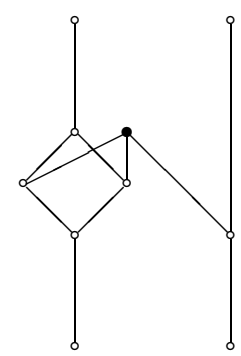

(3.6)

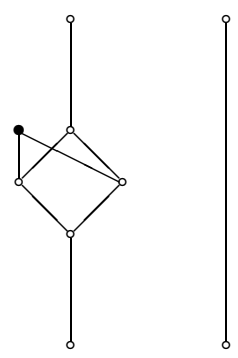

(3.9)

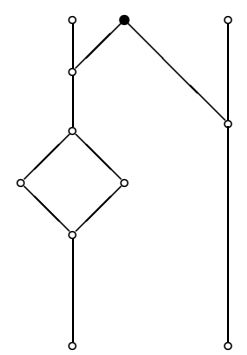

We consider each of cases (3.1)-(3.10) separately. Note that the chain $A=\left\{a_{1} \prec a_{2} \prec \ldots \prec a_{p}\right\}$ is a part of an almost chain located below the unique pair of incomparable elements $a$ and $b$, the chain $B=\left\{b_{1} \prec b_{2} \prec \ldots \prec\right.$ $\left.b_{q}\right\}$ is a part of an almost chain located above the elements $a$ and $b$, and the chain $C=\left\{c_{1} \prec c_{2} \prec \ldots \prec c_{r}\right\}$ is located in the figures to the right.

(3.1) For $r=0$, we have case (3). Let $r \neq 3$. If $p \geq 2$, then we delete the point $a_{p}$. If $q \geq 2$, then we delete the point $b_{q}$. If $r \geq 2$, then we delete the point $c_{r}$. In the three cases considered, we obtain a subset of $n-1$ points that belongs to $\overline{\mathcal{P}}$, which contradicts the conditions of the proposition.

(3.2), (3.3), (3.7), (3.8), (3.10) If $p \geq 2$, then we delete the point $a_{p}$. If $q \geq 2$, then we delete the point $b_{q}$. If $r \geq 2$, then we delete the point $c_{r}$. In each case considered, we obtain a subset of $n-1$ points that belongs to $\overline{\mathcal{P}}$, which contradicts the conditions of the proposition.

(3.4) For $s=q$, we have case (3). Let $s \neq q$. If $p \geq 2$, then we delete the point $a_{p}$. If $q \geq 2$, then we delete the point $b_{q}$. If $r \geq 2$, then we delete the point $c_{r}$. In the three cases considered, we obtain a subset of $n-1$ points that belongs to $\overline{\mathcal{P}}$, which contradicts the conditions of the proposition.

(3.5) If $s=r$ (in particular, $r=0$ ), then we have case (3). Let $s \neq r$. If $p \geq 2$, then we delete the point $a_{p}$. If $q \geq 2$, then we delete the point $b_{q}$. If $r \geq 2$, then we delete the point $c_{r}$. In the three cases considered, we obtain a subset of $n-1$ points that belongs to $\overline{\mathcal{P}}$, which contradicts the conditions of the proposition. 
(3.6) For $q=0$, we have case (3). Let $q \neq 0$. If $p \geq 2$, then we delete the point $a_{p}$. If $q \geq 2$, then we delete the point $b_{q}$. If $r \geq 2$, then we delete the point $c_{r}$. In the three cases considered, we obtain a subset of $n-1$ points that belongs to $\overline{\mathcal{P}}$, which contradicts the conditions of the proposition.

(3.9) If $r=0$ and $s=q$, then we obtain case (3). In the other cases, if $p \geq 2$, then we delete the point $a_{p}$. If $q \geq 2$, then we delete the point $b_{q}$. If $r \geq 2$, then we delete the point $c_{r}$. In the three cases considered, we obtain a subset of $n-1$ points that belongs to $\overline{\mathcal{P}}$, which contradicts the conditions of the proposition.

Thus, we have considered all possible cases and proved that a poset of $n>8$ points belongs to $\mathcal{P}$ if any subset of $n-1$ points in it belongs to $\mathcal{P}$. The proposition is proved.

\section{REFERENCES}

1. V. M. Bondarenko and A. M. Polishchuk, "On a criterion for the positive definiteness of one class of infinite quadratic forms," Nelin. Kolyvannya, 6, No. 1, 3-14 (2003).

2. M. Alsina and P. Bayer, Quaternion Orders, Quadratic Forms, and Shimura Curves, American Mathematical Society, Providence, RI (2004).

3. G. Shimura, Arithmetic and Analytic Theories of Quadratic Forms and Clifford Groups, American Mathematical Society, Providence, RI (2004).

4. D. W. Hoffmann and A. Lanhribi, "Quadratic forms and Pfister neighbors in characteristic 2," Trans. Amer. Math. Soc., No. 10, 40194052 (2004).

5. A. M. Ateiwi, "A study of dichotomy of linear systems of difference equations using the quadratic forms," J. Fract. Calc., 25, 93-100 (2004).

6. F. Fang and J. Pan, "Secondary Brown-Kervaire quadratic forms and $\pi$-manifolds," Forem Math., 16, No. 4, 459-481 (2004).

7. T. Ueno, "Modular forms arising from zeta functions in two variables attached to prehomogeneous vector spaces related to quadratic forms," Nagoya Math. J., 175, 1-37 (2004).

8. W. K. Chan and M. Peters, "Quaternary quadratic forms and Hilbert modular surfaces," Contemp. Math., 344, 85-97 (2004).

9. W. Kohnen, "Special Siegel modular forms and singular series polynomials of quadratic forms," Contemp. Math., 344, 229-236 (2004).

10. A. Laghribi, "Quasi-hyperbolicity of totally singular quadratic forms," Contemp. Math., 344, 237-248 (2004).

11. R. Schulze-Pillot, "Representation by integral quadratic forms—a survey," Contemp. Math., 344, 303-321 (2004).

12. R. W. Fitzgerald and J. L. Yucas, "Pencils of quadratic forms over finite fields," Discrete Math., 283, 71-79 (2004).

13. M. Li and C. Dezhong, "Systems of Hermitian quadratic forms," Can. Math. Bull., 47, No. 1, 73-81 (2004).

14. M. Car, "Quadratic forms with polynomial coefficients," Acta Arithm., 113, No. 2, 131-155 (2004).

15. A. J. Bevelacqua, "Four-dimensional quadratic forms over $F(X)$ where $I_{t}^{3} F(X)=0$ and a failure of the strong Hasse principle," Commun. Alg., 32, No. 3, 855-877 (2004).

16. A. Teksan, "Representations of positive integers by a direct sum of quadratic forms," Results Math., 46, 146-163 (2004).

17. S. Jaschke, C. Keúppelberg, and A. Lindner, "Asymptotic behavior of tails and quantiles of quadratic forms of Gaussian vectors," J. Multivar. Anal., 88, No. 2, 252-273 (2004).

18. P. Gabriel, "Unzerlegbare Darstellungen," Manuscr. Math., 6, 71-103, 309 (1972).

19. S. Brenner, "Quivers with commutativity conditions and some phenomenology of forms," in: Proceedings of the International Conference on Representations of Algebras, No. 5, Carleton University, Ottawa, Ontario (1974).

20. Yu. A. Drozd, "Coxeter transformations and representations of posets," Funkts. Anal. Prilozhen., 8, 34-42 (1974).

21. M. M. Kleiner and A. V. Roiter, "Representations of differential graded categories," in: Yu. A. Mitropol'skii (editor), Matrix Problems [in Russian], Institute of Mathematics, Ukrainian Academy of Sciences, Kiev (1977), pp. 5-70.

22. L. A. Nazarova and A. V. Roiter, "Representations of posets," Zap. Nauchn. Sem. Leningrad. Otdel. Mat. Inst. Akad. Nauk SSSR, 28, 5-31 (1972).

23. V. M. Bondarenko and A. M. Polishchuk, "On subcategories of finite rank of the category of representations of an unbounded poset," Nauk. Visn. Uzhhorod Univ., Issue 8, 15-22 (2003).

24. V. M. Bondarenko and A. M. Polishchuk, "On a Tits quadratic form for infinite posets," Nauk. Visn. Uzhhorod Univ., Issue 7, 28-31 (2002).

25. V. M. Bondarenko, “ On one conjecture for positive-definite quadratic forms," Visn. Kyiv Univ., Ser. Fiz.-Mat. Nauk, No. 3, 11-13 (2005). 